\title{
THE IDEAL-BASED ZERO-DIVISOR GRAPH OF COMMUTATIVE CHAINED RINGS
}

\author{
DAVID F. ANDERSON, S. EBRAHIMI ATANI, M. SHAJARI KOHAN, AND Z. \\ EBRAHIMI SARVANDI
}

\begin{abstract}
Let $I$ be a proper ideal of a commutative ring $R$ with $1 \neq 0$. The ideal-based zero-divisor graph of $R$ with respect to $I$, denoted by $\Gamma_{I}(R)$, is the (simple) graph with vertices $\{x \in R \backslash I \mid x y \in I$ for some $y \in R \backslash I\}$, and distinct vertices $x$ and $y$ are adjacent if and only if $x y \in I$. In this paper, we study $\Gamma_{I}(R)$ for commutative rings $R$ such that $R / I$ is a chained ring.
\end{abstract}

\section{INTRODUCTION}

In the literature, there are many papers on assigning a graph to a ring (see, for example, [1] - [7], [9], and [11]). Among the most interesting graphs are zero-divisor graphs, because they involve both ring theory and graph theory. By studying these graphs, we can gain a broader insight into the concepts and properties that involve both graphs and rings. The concept of zero-divisor graph for a commutative ring $R$ was introduced by I. Beck [7], where he was mainly interested in colorings. In his work, all elements of $R$ were vertices of the graph, and distinct vertices $x$ and $y$ were adjacent if and only if $x y=0$. This investigation of colorings of a commutative ring was then continued by D. D. Anderson and M. Naseer in [1]. Let $Z(R)$ be the set of zero-divisors of $R$. In [5], D. F. Anderson and P. S. Livingston associated a (simple) graph $\Gamma(R)$ to $R$, with vertices $Z(R)^{*}=Z(R) \backslash\{0\}$, the set of nonzero zero-divisors of $R$, and distinct vertices $x$ and $y$ are adjacent if and only if $x y=0$. The zero-divisor graph $\Gamma(R)$ of $R$ has been studied extensively; see the the survey articles [2] and [9].

Let $R$ be a commutative ring with $1 \neq 0, I$ a proper ideal of $R$, and $Z_{I}(R)=\{x \in R \mid x y \in I$ for some $y \in R \backslash I\}$. In [11], S. P. Redmond introduced the ideal-based zero-divisor graph of $R$ with respect to $I$, denoted by $\Gamma_{I}(R)$, with vertices $Z_{I}(R)^{*}=Z_{I}(R) \backslash I=\{x \in R \backslash I \mid x y \in I$ for some

2000 Mathematics Subject Classification. 05C25, 05C38, 13A15.

Key words and phrases. Zero-divisor graph, deal-based zero-divisor graph, chained ring. 
$y \in R \backslash I\}$, and distinct vertices $x$ and $y$ are adjacent if and only if $x y \in I$. Thus $\Gamma_{\{0\}}(R)=\Gamma(R)$ and $\Gamma_{I}(R)$ is the empty graph if and only if $I$ is a prime ideal of $R$. In [11], he explored the relationship between $\Gamma_{I}(R)$ and $\Gamma(R / I)$ and showed, among other things, that $\Gamma_{I}(R)$ is connected with $\operatorname{diam}\left(\Gamma_{I}(R)\right) \in\{0,1,2,3\}$ and $\operatorname{gr}\left(\Gamma_{I}(R)\right) \in\{3,4, \infty\}$.

In [3], D. F. Anderson and A. Badawi studied $\Gamma(R)$ for several classes of rings which generalize valuation domains to the context of rings with zero-divisors. These rings include chained rings and rings $R$ whose prime ideals contained in $Z(R)$ are linearly ordered. Recall that a ring $R$ is a chained ring if the (principal) ideals of $R$ are linearly ordered (by inclusion), equivalently, if either $x \mid y$ or $y \mid x$ for all $x, y \in R$. Examples of chained rings include valuation domains and factor rings of chained rings.

In this paper, we study $\Gamma_{I}(R)$ for commutative rings $R$ such that $R / I$ is a chained ring. Clearly, $R / I$ is a chained ring when $R$ is a chained ring; however, $R / I$ may be a chained ring when $R$ is not a chained ring. For example, let $J$ be a proper ideal of a chained ring $S$ (e.g., a valuation domain), $R=S[X]$ or $S[[X]]$, and $I=(J, X)$. Then $R$ is not a chained ring, but $R / I \cong S / J$ is a chained ring. As another example, let $R_{1}$ and $R_{2}$ be chained rings and $R=R_{1} \times R_{2}$ with ideals $I_{1}=R_{1} \times\{0\}$ and $I_{2}=\{0\} \times R_{2}$. Then $R$ is not a chained ring, but $R / I_{1} \cong R_{2}$ and $R / I_{2} \cong R_{1}$ are both chained rings.

In Section 2, we study the relationship between several natural subgraphs of $\Gamma_{I}(R)$. Then, in Section 3, we specialize to the case when $R / I$ is a chained ring. We completely characterize the diameter and girth of the graph $\Gamma_{I}(R)$ for such rings in Theorem 3.8 and Theorem 3.9, respectively. Moreover, we extend several results in [3] to the more general ideal-based zero-divisor graph case. In fact, results in [3] for $\Gamma(R)$ when $R$ is a chained ring are actually special cases of the results in this paper for $\Gamma_{I}(R)$ when $R / I$ is a chained ring since if $I=\{0\}$, then $R$ is a chained ring and $\Gamma(R)=\Gamma_{I}(R)$. We invite the interested reader to compare the results in [3] for $\Gamma(R)$ to the results in this paper for $\Gamma_{I}(R)$.

In order to make this paper easier to follow, we next recall various notions which will be used in the sequel. For a graph $\Gamma$, let $E(\Gamma)$ and $V(\Gamma)$ denote the sets of edges and vertices of $\Gamma$, respectively. By abuse of notation, we will often refer to a subgraph of $\Gamma_{I}(R)$ by its set of vertices; all such subgraphs will be induced subgraphs. We recall that a graph is connected if there exists a path connecting any two distinct vertices. At the other extreme, we say that a graph $\Gamma$ is totally disconnected if no two vertices of $\Gamma$ are adjacent. The distance between two distinct vertices $a$ and $b$ in $\Gamma$, denoted by $d(a, b)$, is the length of a shortest path connecting them $(d(a, a)=0$ and $d(a, b)=\infty$ if there is no such path). The diameter of a graph $\Gamma$, denoted by 
$\operatorname{diam}(\Gamma)$, is $\sup \{d(a, b) \mid a, b \in V(\Gamma)\}$. A graph is complete if it is connected with diameter less than or equal to one. The girth of a graph $\Gamma$, denoted by $\operatorname{gr}(\Gamma)$, is the length of a shortest cycle in $\Gamma$, provided $\Gamma$ contains a cycle; otherwise, $\operatorname{gr}(\Gamma)=\infty$. Recall that a graph $\Gamma$ is a star graph if it has a vertex that is adjacent to every other vertex and this is the only adjacency relation. Throughout this paper, all rings are assumed to be commutative with $1 \neq 0$. As usual, $\mathbb{Z}, \mathbb{Z}_{n}$, and $\mathbb{Q}$ denote the rings of integers, integers modulo $n$, and rational numbers, respectively; for an ideal $I$ of $R, \sqrt{I}=\left\{x \in R \mid x^{n} \in I\right.$ for some integer $n \geq 1\}$; and $\operatorname{nil}(R)=\sqrt{\{0\}}$. To avoid any trivalities when $\Gamma_{I}(R)$ is the empty graph, we will implicitly assume when necessary that $I$ is not a prime ideal of $R$. For a ring theory reference, see [10]; for a graph theory reference, see [8].

\section{SubGraphs OF $\Gamma_{I}(R)$}

Let $I$ be a proper ideal of a commutative ring $R$. In this section, we investigate the relationship between several subgraphs of $\Gamma_{I}(R)$. It will be convenient to let $Z_{I}(R)^{*}=Z_{I}(R) \backslash I=\{x \in R \backslash I \mid x y \in I$ for some $y \in R \backslash I\}$. Note that $Z_{\{0\}}(R)=Z(R), Z(R / I)=Z_{I}(R) / I$, and $V\left(\Gamma_{I}(R)\right)=$ $Z_{I}(R)^{*}$. Moreover, $Z_{I}(R)^{*}=\emptyset$ (i.e., $\left.Z_{I}(R)=I\right)$ if and only if $I$ is a prime ideal of $R$. Also, let $N_{I}(R)=\left\{x \in R \mid x^{2} \in I\right\}$ and $N_{I}(R)^{*}=N_{I}(R) \backslash I$. Clearly, $I \subseteq N_{I}(R) \subseteq \sqrt{I}$, and $N_{I}(R)^{*}=\emptyset$ (i.e., $N_{I}(R)=I$ ) if and only if $I$ is a radical ideal of $R$ (i.e., $\sqrt{I}=I$ ).

Proposition 2.1. Let $I$ be a proper ideal of a commutative ring $R, \sqrt{I}^{*}=$ $\sqrt{I} \backslash I, Z_{I}(R)=\{x \in R \mid x y \in I$ for some $y \in R \backslash I\}, Z_{I}(R)^{*}=Z_{I}(R) \backslash I$, and $N_{I}(R)=\left\{x \in R \mid x^{2} \in I\right\}$. Then the following hold.

(1) $\sqrt{I}^{*} \subseteq Z_{I}(R)^{*}$.

(2) $I \subseteq N_{I}(R) \subseteq \sqrt{I} \subseteq Z_{I}(R)$.

(3) If $Z_{I}(R)$ is an ideal of $R$, then it is a prime ideal of $R$.

(4) $N_{I}(R)=I$ if and only if $\sqrt{I}=I$.

Proof. (1) Let $x \in \sqrt{I}^{*}=\sqrt{I} \backslash I$. Let $n(n \geq 2)$ be the least positive integer such that $x^{n} \in I$. As $x \notin I, x^{n-1} \notin I$, and $x x^{n-1}=x^{n} \in I$, we have $x \in Z_{I}(R) \backslash I=Z_{I}(R)^{*}$.

(2) This follows from part (1) and the above comments.

(3) Suppose that $Z_{I}(R)$ is an ideal of $R$, and let $x, y \in R$ such that $x y \in Z_{I}(R)$. Then there is a $z \in R \backslash I$ such that $(x y) z \in I$. If $y z \in I$, then $y \in Z_{I}(R)$. If $y z \notin I$, then $x \in Z_{I}(R)$. Thus $Z_{I}(R)$ is a prime ideal of $R$. (This also follows since $Z_{I}(R)$ is a union of prime ideals of $R$ ).

(4) This is clear. 
Theorem 2.2. Let $I$ be a proper ideal of a commutative ring $R, \sqrt{I}^{*}=\sqrt{I} \backslash$ $I, Z_{I}(R)=\{x \in R \mid x y \in I$ for some $y \in R \backslash I\}$, and $Z_{I}(R)^{*}=Z_{I}(R) \backslash I$. Then the following hold.

(1) If $x \in \sqrt{I}^{*}$ and $y \in Z_{I}(R)^{*}$, then $d(x, y) \leq 2$ in $\Gamma_{I}(R)$.

(2) The subgraph $Z_{I}(R) \backslash \sqrt{I}$ of $\Gamma_{I}(R)$ is totally disconnected if and only if $\sqrt{I}$ is a prime ideal of $R$.

Proof. (1) We may assume that $x \neq y$ and $x y \notin I$. Since $y \in Z_{I}(R) \backslash I$ and $x y \notin I$, there is a $z \in Z_{I}(R) \backslash(I \cup\{x\})$ such that $z y \in I$. There is a least positive integer $n$ such that $x^{n} z \in I$ since $x \in \sqrt{I}^{*}$. If $n=1$, then $x-z-y$ is a path of length 2 from $x$ to $y$. If $n \geq 2$, then $x-x^{n-1} z-y$ is a path of length 2 from $x$ to $y$. Thus $d(x, y) \leq 2$ in $\Gamma_{I}(R)$.

(2) Assume that $\sqrt{I}$ is a prime ideal of $R$, and let $x$ and $y$ be distinct elements of $Z_{I}(R) \backslash \sqrt{I}$. If $x$ and $y$ are adjacent, then $x y \in I \subseteq \sqrt{I}$. Thus either $x$ or $y$ belongs to $\sqrt{I}$, a contradiction. Hence the subgraph $Z_{I}(R) \backslash \sqrt{I}$ is totally disconnected.

Conversely, assume that $\sqrt{I}$ is not a prime ideal of $R$. Then there are $x, y \in R \backslash \sqrt{I}$ with $x y \in \sqrt{I}$. Thus $x^{n} y^{n}=(x y)^{n} \in I$ for some positive integer $n$. If $x^{n}=y^{n}$, then $x^{2 n}=x^{n} y^{n} \in I$; so $x \in \sqrt{I}$, a contradiction. Hence $x^{n}, y^{n} \in Z_{I}(R) \backslash \sqrt{I}, x^{n} \neq y^{n}$, and $x^{n}$ and $y^{n}$ are adjacent. Thus the subgraph $Z_{I}(R) \backslash \sqrt{I}$ is not totally disconnected.

Proposition 2.3. Let $I$ be a proper ideal of a commutative ring $R$ and $N_{I}(R)=\left\{x \in R \mid x^{2} \in I\right\}$. Then every vertex of the subgraph $\sqrt{I} \backslash N_{I}(R)$ of $\Gamma_{I}(R)$ is adjacent to a vertex of the subgraph $N_{I}(R)^{*}=N_{I}(R) \backslash I$ of $\Gamma_{I}(R)$.

Proof. Let $x \in \sqrt{I} \backslash N_{I}(R), n(n \geq 3)$ be the least positive integer such that $x^{n} \in I$, and $y=x^{n-1}$. Then $y=x^{n-1} \notin I, x y=x x^{n-1}=x^{n} \in I$, and $y^{2}=\left(x^{n-1}\right)^{2}=x^{2 n-2} \in I$ since $2 n-2 \geq n$ as $n \geq 3$. Thus $y \in N_{I}(R) \backslash I=$ $N_{I}(R)^{*}, x \neq y$, and $x$ is adjacent to $y$ in $\Gamma_{I}(R)$ since $x y \in I$.

Thus $\Gamma_{I}(R)$ is the union of three, possibly empty, disjoint subgraphs, $N_{I}(R)^{*}=N_{I}(R) \backslash I, \sqrt{I} \backslash N_{I}(R)$, and $Z_{I}(R) \backslash \sqrt{I}$. Suppose that the ideal $I$ is not a prime ideal of $R$, but $\sqrt{I}$ is a prime ideal of $R$. Then $N_{I}(R)^{*}$ is nonempty by Proposition $2.1(4)$ and $Z_{I}(R) \backslash \sqrt{I}$ is totally disconnected by Theorem $2.2(2)$.

\section{Chained Rings}

In this section, we investigate the ideal-based zero-divisor graph $\Gamma_{I}(R)$ with respect to a proper ideal $I$ of a commutative $\operatorname{ring} R$ such that $R / I$ is a chained ring. In particular, these results all hold when $R$ is a chained ring. 
Note that $\sqrt{I}$ is a prime ideal of $R$ when $R / I$ is a chained ring since radical ideals in chained rings are prime ideals.

We first show, among other things, that every vertex of the subgraph $Z_{I}(R) \backslash N_{I}(R)$ is adjacent to a vertex of the subgraph $N_{I}(R)^{*}=N_{I}(R) \backslash I$ and every two distinct vertices of $N_{I}(R)^{*}$ are adjacent (i.e., $N_{I}(R)^{*}$ is a complete subgraph of $\Gamma_{I}(R)$ ).

Proposition 3.1. Let $I$ be a proper ideal of a commutative ring $R$ such that $R / I$ is a chained ring, $Z_{I}(R)=\{x \in R \mid x y \in I$ for some $y \in R \backslash I\}$, $Z_{I}(R)^{*}=Z_{I}(R) \backslash I, N_{I}(R)=\left\{x \in R \mid x^{2} \in I\right\}, N_{I}(R)^{*}=N_{I}(R) \backslash I$, and $x, y \in R$.

(1) If $x y \in I$, then either $x \in N_{I}(R)$ or $y \in N_{I}(R)$.

(2) If $x, y \in N_{I}(R)$, then $x y \in I$.

(3) If $x, y \in Z_{I}(R) \backslash N_{I}(R)$, then $x y \notin I$.

(4) If $x \in Z_{I}(R)^{*}$, then $x y \in I$ for some $y \in N_{I}(R)^{*}$.

(5) If $x_{1}, \ldots, x_{n} \in Z_{I}(R)^{*}$, then there is a $y \in N_{I}(R)^{*}$ such that $x_{i} y \in I$ for every integer $i, 1 \leq i \leq n$.

(6) $N_{I}(R)$ is an ideal of $R$. Moreover, $N_{I}(R)=I$ if and only if $I$ is a prime ideal of $R$.

(7) $N_{I}(R)$ is a prime ideal of $R$ if and only if $N_{I}(R)=\sqrt{I}$.

(8) $Z_{I}(R)$ is a prime ideal of $R$.

Proof. (1) Since $R / I$ is a chained ring, we may assume that $(x+I) \mid(y+I)$ in $R / I$. Thus $y=a x+i$ for some $a \in R$ and $i \in I$. Hence $y^{2}=(a x+i) y=$ $a x y+i y \in I$ since $x y \in I$; so $y \in N_{I}(R)$.

(2) Since $R / I$ is a chained ring, we may assume that $(x+I) \mid(y+I)$ in $R / I$. Thus $y=a x+i$ for some $a \in R$ and $i \in I$. Hence $x y=x(a x+i)=$ $a x^{2}+x i \in I$ since $x^{2} \in I$.

(3) This follows from part (1) above.

(4) If $x \in N_{I}(R)^{*}$, then let $y=x$. If $x \in Z_{I}(R) \backslash N_{I}(R)$, then there is a $y \in R \backslash I$ such that $x y \in I$. By part (3) above, we have $y \in N_{I}(R)^{*}$.

(5) Since $R / I$ is a chained ring, there is an integer $j, 1 \leq j \leq n$, such that $\left(x_{j}+I\right) \mid\left(x_{i}+I\right)$ for every integer $i, 1 \leq i \leq n$. Thus $x_{i}=a_{i} x_{j}+b_{i}$ for some $a_{i} \in R$ and $b_{i} \in I$ for every integer $i, 1 \leq i \leq n$. By part (4) above, there is a $y \in N_{I}(R)^{*}$ such that $x_{j} y \in I$. Hence $x_{i} y=\left(a_{i} x_{j}+b_{i}\right) y=a_{i} x_{j} y+b_{i} y \in I$ for every integer $i, 1 \leq i \leq n$.

(6) Let $x, y \in N_{I}(R)$ and $r \in R$. Then $(r x)^{2}=r^{2} x^{2} \in I$ since $x^{2} \in I$; so $r x \in N_{I}(R)$. Thus we need only show that $x+y \in N_{I}(R)$. By assumption, $x^{2}, y^{2} \in I$, and $x y \in I$ by part (2) above; so $(x+y)^{2}=x^{2}+2 x y+y^{2} \in$ $I$. Hence $N_{I}(R)$ is an ideal of $R$. The "moreover" statement follows from Proposition 2.1 (4) since $I$ is a prime ideal of $R$ if and only if $I$ is a radical ideal of $R$ as $R / I$ is a chained ring. 
(7) Suppose that $N_{I}(R)$ is a prime ideal of $R$. Then $N_{I}(R)=\sqrt{I}$ since $I \subseteq N_{I}(R) \subseteq \sqrt{I}$. Conversely, assume that $N_{I}(R)=\sqrt{I}$. Then $\sqrt{I}$ is a prime ideal of $R$ since $R / I$ is a chained ring.

(8) Since $Z_{I}(R)$ is closed under multiplication and by Proposition 2.1 (3), we need only show that $Z_{I}(R)$ is closed under addition. Let $x, y \in Z_{I}(R)$. Since $R / I$ is a chained ring, we may assume that $(x+I) \mid(y+I)$ in $R / I$, and thus $y=a x+i$ for some $a \in R$ and $i \in I$. Let $z \in R \backslash I$ such that $x z \in I$. Then $(x+y) z=(x+a x+i) z=(1+a) x z+i z \in I$; so $x+y \in Z_{I}(R)$.

Remark 3.2. If $R / I$ is not a chained ring, then $N_{I}(R)$ need not be an ideal of $R$. For example, let $R=\mathbb{Z}[X, Y]$ and $I=\left(X^{2}, Y^{2}\right)$. Then $X, Y \in N_{I}(R)$, but $X+Y \notin N_{I}(R)$. However, $N_{I}(R)$ is an ideal of $R$ when $\operatorname{char}(R)=2$.

Let $R=\mathbb{Z}_{2}[X, Y]$ and $I=\left(X^{4}, Y^{4}\right)$. Then $R / I$ is not a chained ring, $N_{I}(R)$ is an ideal of $R$ since $\operatorname{char}(R)=2$, and $I \subsetneq\left(X^{2}, Y^{2}\right)=N_{I}(R) \subsetneq$ $(X, Y)=\sqrt{I}=Z_{I}(R)$.

The next result improves Theorem $2.2(2)$ when $R / I$ is a chained ring.

Theorem 3.3. Let $I$ be a proper ideal ideal of a commutative ring $R$ such that $R / I$ is a chained ring, $Z_{I}(R)=\{x \in R \mid x y \in I$ for some $y \in R \backslash I\}$, $N_{I}(R)=\left\{x \in R \mid x^{2} \in I\right\}$, and $N_{I}(R)^{*}=N_{I}(R) \backslash I$. Then $N_{I}(R)^{*}$ is a complete subgraph of $\Gamma_{I}(R)$ and the subgraph $Z_{I}(R) \backslash N_{I}(R)$ of $\Gamma_{I}(R)$ is totally disconnected. Moreover, $N_{I}(R)^{*}$ is nonempty if and only if $\Gamma_{I}(R)$ is nonempty.

Proof. The first statement follows from parts (2) and (3) of Proposition 3.1, respectively. The "moreover" statement follows since $N_{I}(R)^{*}=\emptyset$ (i.e., $\left.N_{I}(R)=I\right)$ if and only if $\sqrt{I}=I$ by Proposition $2.1(4)$, if and only if $I$ is a prime ideal of $R$ (since $R / I$ is a chained ring), if and only if $Z_{I}(R)^{*}=\emptyset$ (i.e., $\Gamma_{I}(R)$ is the empty graph).

Corollary 3.4. Let $I$ be a proper ideal ideal of a commutative ring $R$ such that $R / I$ is a chained ring. Then $\Gamma_{I}(R)$ is a complete graph if and only if $Z_{I}(R)=N_{I}(R)$. Moreover, if $\Gamma_{I}(R)$ is a complete graph, then $Z(R / I)=$ $\operatorname{nil}(R / I)$.

Proof. We first show that $\Gamma_{I}(R)$ is complete if and only if $Z_{I}(R)=N_{I}(R)$. If $Z_{I}(R)=N_{I}(R)$, then $\Gamma_{I}(R)$ is complete by Theorem 3.3. Conversely, suppose that $N_{I}(R) \subsetneq Z_{I}(R)$. Let $x \in Z_{I}(R) \backslash N_{I}(R)$. Then $x y \in I$ for some $y \in N_{I}(R)^{*}=N_{I}(R) \backslash I$ by Proposition 3.1 (4), and thus $x+y \in Z_{I}(R)$ by Proposition 3.1 (8). Moreover, $x+y \notin N_{I}(R)$ since $y \in N_{I}(R), x \notin N_{I}(R)$, and $N_{I}(R)$ is an ideal of $R$ by Proposition $3.1(6)$. Hence $x$ and $x+y$ are distinct, nonadjacent vertices since $Z_{I}(R) \backslash N_{I}(R)$ is totally disconnected by Theorem 3.3. Hence $\Gamma_{I}(R)$ is not complete. 
For the "moreover" statement, suppose that $\Gamma_{I}(R)$ is a complete graph. Then $Z_{I}(R)=N_{I}(R)$ by above, and thus $Z_{I}(R)=\sqrt{I}$ by Proposition 2.1 (2). Hence nil $(R / I)=\sqrt{I} / I=Z_{I}(R) / I=Z(R / I)$.

Remark 3.5. (1) Corollary 3.4 also follows from [4, Theorem 4.6] and [6, Theorem 4.7]. Note that the converse of the "moreover" statement in Corollary 3.4 need not hold. For example, let $R=\mathbb{Z}_{2} \times \mathbb{Z}_{8}$ and $I=\mathbb{Z}_{2} \times\{0\}$. Then $R / I \cong \mathbb{Z}_{8}$ is a finite local ring; so $\operatorname{nil}(R / I)=Z(R / I)$. However, $\Gamma_{I}(R)$ is not complete (cf. Example 3.10). Also, Corollary 3.4 may fail if $R / I$ is not a chained ring. For example, let $R=\mathbb{Z}_{2} \times \mathbb{Z}_{2}$ and $I=\{(0,0)\}$. Then $\Gamma_{I}(R)=\Gamma(R)$ is the complete graph on two vertices, but $N_{I}(R)=I \subsetneq$ $R \backslash\{(1,1)\}=Z_{I}(R)$.

(2) Let $I$ be a proper ideal of a commutative ring $R$ such that $R / I$ is a chained ring. Note that if $\sqrt{I} \subsetneq Z_{I}(R)$, then $R / I$ is infinite. This follows since if $R / I$ is finite, then $\sqrt{I} / I$ is a prime, hence maximal, ideal of $R / I$ contained in the prime ideal $Z_{I}(R) / I$; so $\sqrt{I}=Z_{I}(R)$. Moreover, if $\Gamma_{I}(R)$ is an infinite graph (i.e., $I$ is not a prime ideal of $R$ and either $I$ is infinite or $R / I$ is infinite), then the subgraph $Z_{I}(R) \backslash N_{I}(R)$ is infinite if it is nonempty. This is clear if $N_{I}(R)$ is finite. If $N_{I}(R)$ is infinite, it follows since $x+$ $N_{I}(R) \subseteq Z_{I}(R) \backslash N_{I}(R)$ for $x \in Z_{I}(R) \backslash N_{I}(R)$.

(3) Let $I$ be a proper ideal of a commutative ring $R$ such that $R / I$ is a chained ring. Then there are eight possibilities for equals or strict inclusion in the chain of ideals $I \subseteq N_{I}(R) \subseteq \sqrt{I} \subseteq Z_{I}(R)$ (i.e., for the subgraphs $N_{I}(R) \backslash I, \sqrt{I} \backslash N_{I}(R)$, and $Z_{I}(R) \backslash \sqrt{I}$ of $\Gamma_{I}(R)$ being empty or nonempty). If $N_{I}(R)=I$, then $I$ is a prime ideal of $R$ by Proposition 3.1 (6); so in this case, all four ideals are equal and $\Gamma_{I}(R)$ is the empty graph. Easy examples show that the other four cases are all possible. For example, let $R=\mathbb{Z}_{(2)}+X \mathbb{Q}[[X]]$ and $I=\left(X^{2}\right)$. Then $R$ is a valuation domain; so $R / I$ is a chained ring. Note that $I \subsetneq(X)=N_{I}(R) \subsetneq X \mathbb{Q}[[X]]=\sqrt{I} \subsetneq(2)=Z_{I}(R)$.

When $R / I$ is a chained ring, the graph $\Gamma_{I}(R)$ is easy to describe. It is the union of two disjoint subgraphs, $N_{I}(R)^{*}=N_{I}(R) \backslash I$ (nonempty when $\Gamma_{I}(R)$ is nonempty) and $Z_{I}(R) \backslash N_{I}(R)$ (possibly empty), where $N_{I}(R)^{*}$ is complete and $Z_{I}(R) \backslash N_{I}(R)$ is totally disconnected by Theorem 3.3, and every vertex of $Z_{I}(R) \backslash N_{I}(R)$ is adjacent to some vertex of $N_{I}(R)$ by Proposition 3.1(4).

Recall that $\operatorname{diam}\left(\Gamma_{I}(R)\right) \in\{0,1,2,3\}$ and $\operatorname{gr}\left(\Gamma_{I}(R)\right) \in\{3,4, \infty\}$ for every proper ideal $I$ of a commutative ring $R$. Stronger results hold for the diameter and girth of $\Gamma_{I}(R)$ when $R / I$ is a chained ring.

Theorem 3.6. Let $I$ be a proper ideal of a commutative ring $R$ such that $R / I$ is a chained ring. Then $\operatorname{diam}\left(\Gamma_{I}(R)\right) \in\{0,1,2\}$.

Proof. Let $Z_{I}(R)^{*}=Z_{I}(R) \backslash I=V\left(\Gamma_{I}(R)\right)$ and $N_{I}(R)=\left\{x \in R \mid x^{2} \in I\right\}$. If $\left|Z_{I}(R)^{*}\right| \leq 1$, then $\operatorname{diam}\left(\Gamma_{I}(R)\right)=0$. So we may assume that $\left|Z_{I}(R)^{*}\right| \geq 2$. 
Let $x, y \in Z_{I}(R)^{*}$ with $x \neq y$. If $x, y \in N_{I}(R)$, then $x y \in I$ by Proposition 3.1 (2), and thus $d(x, y)=1$. If $x \in N_{I}(R)$ and $y \notin N_{I}(R)$, then $y z \in I$ for some $z \in N_{I}(R)^{*} \subseteq Z_{I}(R)^{*}$ by Proposition 3.1 (4) and $x z \in I$ by Proposition $3.1(2)$. If $x=z$, then $d(x, y)=1$. Otherwise, $x-z-y$ is a path of length 2 from $x$ to $y$, and hence $d(x, y) \leq 2$. Finally, let $x, y \notin N_{I}(R)$. Then $x z, y z \in I$ for some $z \in N_{I}(R)^{*} \subseteq Z_{I}(R)^{*}$ by Proposition 3.1 (5). Thus $x-z-y$ is a path of length 2 from $x$ to $y$, and hence $d(x, y) \leq 2$ (actually, $d(x, y)=2$ since $x y \notin I$ by Proposition 3.1(3)). Thus $\operatorname{diam}\left(\Gamma_{I}(R)\right) \in\{0,1,2\}$.

Remark 3.7. $\operatorname{diam}\left(\Gamma_{I}(R)\right)=0$ (i.e., $\left|Z_{I}(R)^{*}\right| \leq 1$ ) if and only if either $\Gamma_{I}(R)$ is the empty graph (i.e., $I$ is a prime ideal of $R$ ) or $I=\{0\}$ (i.e., $\left.\Gamma_{I}(R)=\Gamma(R)\right)$ and $R \cong \mathbb{Z}_{4}$ or $\mathbb{Z}_{2}[X] /\left(X^{2}\right)$, both of which are chained rings.

Next, we explicitly determine when the diameter of $\Gamma_{I}(R)$ is either 0,1 , or 2 .

Theorem 3.8. Let $I$ be a proper ideal of a commutative ring $R$ such that $R / I$ is a chained ring, $Z_{I}(R)=\{x \in R \mid x y \in I$ for some $y \in R \backslash I\}$, $Z_{I}(R)^{*}=Z_{I}(R) \backslash I$, and $N_{I}(R)=\left\{x \in R \mid x^{2} \in I\right\}$. Then exactly one of the following three cases must occur.

(1) $\left|Z_{I}(R)^{*}\right| \leq 1$. In this case, $\operatorname{diam}\left(\Gamma_{I}(R)\right)=0$.

(2) $\left|Z_{I}(R)^{*}\right| \geq 2$ and $N_{I}(R)=Z_{I}(R)$. In this case, $\operatorname{diam}\left(\Gamma_{I}(R)\right)=1$.

(3) $\left|Z_{I}(R)^{*}\right| \geq 2$ and $N_{I}(R) \subsetneq Z_{I}(R)$. In this case, $\operatorname{diam}\left(\Gamma_{I}(R)\right)=2$.

Proof. This follows directly from Proposition 3.1 and the proof of Theorem 3.6.

We next show that $\operatorname{gr}\left(\Gamma_{I}(R)\right) \in\{3, \infty\}$ when $R / I$ is a chained ring.

Theorem 3.9. Let $I$ be a proper ideal of a commutative ring $R$ such that $R / I$ is a chained ring, $Z_{I}(R)=\{x \in R \mid x y \in I$ for some $y \in R \backslash I\}$, $N_{I}(R)=\left\{x \in R \mid x^{2} \in I\right\}$, and $N_{I}(R)^{*}=N_{I}(R) \backslash I$. Then exactly one of the following four cases must occur.

(1) $\left|N_{I}(R)^{*}\right| \leq 1$. In this case, $\operatorname{gr}\left(\Gamma_{I}(R)\right)=\infty$.

(2) $\left|N_{I}(R)^{*}\right|=2$ and $N_{I}(R)=Z_{I}(R)$. In this case, $\operatorname{gr}\left(\Gamma_{I}(R)\right)=\infty$.

(3) $\left|N_{I}(R)^{*}\right|=2$ and $N_{I}(R) \subsetneq Z_{I}(R)$. In this case, $\operatorname{gr}\left(\Gamma_{I}(R)\right)=3$.

(4) $\left|N_{I}(R)^{*}\right| \geq 3$. In this case, $\operatorname{gr}\left(\Gamma_{I}(R)\right)=3$.

Proof. (1) We may assume that $N_{I}(R)^{*} \neq \emptyset$ by the "moreover" statement in Theorem 3.3. Let $N_{I}(R)^{*}=\{x\}$. If $N_{I}(R)^{*}=Z_{I}(R)^{*}$, then $\operatorname{gr}\left(\Gamma_{I}(R)\right)=\infty$. If $N_{I}(R)^{*} \subsetneq Z_{I}(R)^{*}$, then $\Gamma_{I}(R)$ is a star graph with center $x$ by parts (3) and (4) of Proposition 3.1. Thus $\operatorname{gr}\left(\Gamma_{I}(R)\right)=\infty$.

(2) By hypothesis, $\left|Z_{I}(R)^{*}\right|=2$; hence $\operatorname{gr}\left(\Gamma_{I}(R)\right)=\infty$. 
(3) Let $N_{I}(R)^{*}=\{x, y\}$. Then $x y \in I$ by Proposition $3.1(2)$ and $x+y \in$ $N_{I}(R)$ by Proposition 3.1 (6). If $x+y \in N_{I}(R) \backslash I=N_{I}(R)^{*}$, then either $x+y=x$ or $x+y=y$. Thus either $y=0$ or $x=0$, a contradiction. Hence $x+y \in I$. Let $z \in Z_{I}(R) \backslash N_{I}(R)^{*}$. Then either $x z \in I$ or $y z \in I$ by Proposition 3.1 (4). However, in either case, $x z, y z \in I$ since $x+y \in I$. Thus $x-y-z-x$ is a triangle in $\Gamma_{I}(R)$; so $\operatorname{gr}\left(\Gamma_{I}(R)\right)=3$.

(4) If $\left|N_{I}(R)^{*}\right| \geq 3$, then $\operatorname{gr}\left(\Gamma_{I}(R)\right)=3$ by Proposition $3.1(2)$.

The final example illustrates the above results. In particular, it shows that all possible values may be realized for $\operatorname{diam}\left(\Gamma_{I}(R)\right)$ and $\operatorname{gr}\left(\Gamma_{I}(R)\right)$ when $R / I$ is a chained ring and $I$ is a nonzero ideal of $R$. For the $\operatorname{diam}\left(\Gamma_{I}(R)\right)=0$ case, see Remark 3.7.

Example 3.10. Note that $\mathbb{Z}_{n}$ is a chained ring if and only if $n$ is a prime power. Let $p$ be a prime number, and for every positive integer $n$, let $R_{n}=\mathbb{Z}_{2} \times \mathbb{Z}_{p^{n}}$ and $I_{n}=\mathbb{Z}_{2} \times\{0\}$. Then $R_{n} / I_{n} \cong \mathbb{Z}_{p^{n}}$ is a chained ring. It is easily verified (cf. Theorem 3.9) that $\Gamma_{I_{1}}\left(R_{1}\right)$ is the empty graph, $\operatorname{gr}\left(\Gamma_{I_{2}}\left(R_{2}\right)\right)=\infty$ if $p=2, \operatorname{gr}\left(\Gamma_{I_{2}}\left(R_{2}\right)\right)=3$ if $p \neq 2$, and $\operatorname{gr}\left(\Gamma_{I_{n}}\left(R_{n}\right)\right)=3$ for $n \geq 3$ since $(0, p)-\left(1, p^{n-1}\right)-\left(0, p^{n-1}\right)-(0, p)$ is a triangle. It is also easily verified that $\operatorname{diam}\left(\Gamma_{I_{2}}\left(R_{2}\right)\right)=1$ and $\operatorname{diam}\left(\Gamma_{I_{n}}\left(R_{n}\right)\right)=2$ for $n \geq 3$ (cf. Theorem 3.8).

\section{REFERENCES}

[1] D. D. Anderson and M. Naseer, Beck's coloring of commutative rings, J. Algebra, 159 (1993), 500-514.

[2] D. F. Anderson, M. C. Axtell and J. A. Stickles, Jr., Zero-divisor graphs in commutative rings, in Commutative Algebra, Noetherian and Non-Noetherian Perspectives (M. Fontana, S.-E. Kabbaj, B. Olberding, I. Swanson, Eds.), Springer-Verlag, New York, 2011, 23-45.

[3] D. F. Anderson and A. Badawi, On the zero-divisor graph of a commutative ring, Commun. Algebra, 36 (2008), 3073-3092

[4] D. F. Anderson and A. Badawi, The total graph of a commutative ring, J. Algebra, 320 (2008), 2706-2719.

[5] D. F. Anderson and P. S. Livingston, The zero-divisor graph of a commutative ring, J. Algebra, 217 (1999), 434-447.

[6] D. F. Anderson and S. Shirinkam, Some remarks on the graph $\Gamma_{I}(R)$, Commun. Algebra, to appear.

[7] I. Beck, Coloring of commutative rings, J. Algebra, 116 (1988), 208-226.

[8] B. Bolloboás, Modern Graph Theory, Springer, New York, 1998.

[9] J. Coykendall, S. Sather-Wagstaff, L. Sheppardson, and S. Spiroff, On zero divisor graphs, in Progress in Commutative Algebra 2: Closure, Finiteness and Factorization (C. Francisco et al. Eds.), Walter Gruyter, Berlin, 2012, 241-299.

[10] I. Kaplansky, Commutative Rings, Rev. Ed., University of Chicago Press, Chicago, 1974.

[11] S. P. Redmond, An ideal-based zero-divisor graph of a commutative ring, Commun. Algebra, 31 (2003), 4425-4443. 
(Received: July 4, 2013)

(Revised: April 2, 2014)
David F. Anderson

Department of Mathematics

The University of Tennessee

Knoxville, TN 37996

U.S.A.

anderson@math.utk.edu

S. Ebrahimi Atani, M. Shajari Kohan,

Z. Ebrahimi Sarvandi

Department of Mathematics

University of Guilan

P.O. Box 1914, Rasht

Iran

ebrahimiatani@gmail.com 